International Journal of Pure and Applied Mathematics

Volume 109 No. 4 2016, 919-939

ISSN: 1311-8080 (printed version); ISSN: 1314-3395 (on-line version)

url: http://www.ijpam.eu

doi: 10.12732/ijpam.v109i4.15

\title{
COMMON FIXED POINTS FOR MINIMAL COMMUTING MAPPINGS SATISFYING $(\psi-\phi)$-CONTRACTIVE CONDITIONS IN MULTIPLICATIVE METRIC SPACES
}

\author{
Young Chel Kwun ${ }^{1}$, Poonam Nagpal ${ }^{2}$, Sanjay Kumar ${ }^{3}$, \\ Sudhir Kumar Garg ${ }^{4}$, Shin Min Kang ${ }^{5} \S$ \\ ${ }^{1}$ Department of Mathematics \\ Dong-A University \\ Busan, 49315, KOREA \\ ${ }^{2,3,4}$ Departement of Mathematics \\ Deenbandhu Chhotu Ram University of Science and Technology \\ Murthal, Sonepat 131039, Haryana, INDIA \\ ${ }^{5}$ Department of Mathematics and RINS \\ Gyeongsang National University \\ Jinju, 52828, KOREA
}

\begin{abstract}
In this paper, we prove common fixed point theorems for minimal commuting mappings satisfying $(\psi-\phi)$-contractive conditions in multiplicative metric spaces.
\end{abstract}

AMS Subject Classification: $47 \mathrm{H} 10,54 \mathrm{H} 25$

Key Words: multiplicative metric spaces, minimal commuting mappings, altering distances

\section{Introduction and Preliminaries}

The fixed point theory has applications not only in many areas of mathematics

\footnotetext{
Received: $\quad$ August 18, 2016

Revised: $\quad$ September 10, 2016

Published: $\quad$ October 7, 2016

(c) 2016 Academic Publications, Ltd.

url: www.acadpubl.eu

$\S_{\text {Correspondence author }}$
} 
but also in many branches of quantitative sciences such as economics and computer sciences. The most famous result in this field is known as the Banach contraction principle ([4]). Many authors generalized the Banach contraction principle in various spaces such as quasi-metric spaces, fuzzy metric spaces, cone metric spaces, partial metric spaces and generalized metric spaces.

In 2008, Bashirov et al. [5] introduced the notion of multiplicative metric spaces, and studied the concept of multiplicative calculus and proved the fundamental theorem of multiplicative calculus.

In 2012, Florack and Assen [10] displayed the use of the concept of multiplicative calculus in biomedical image analysis. In 2011, Bashirov et al. [6] exploit the efficiency of multiplicative calculus over the Newtonian calculus. They demonstrated that the multiplicative differential equations are more suitable than the ordinary differential equations in investigating some problems in various fields. They defined the multiplicative distance between two nonnegative real numbers as well as between two positive square matrices by using multiplicative absolute value function. This provides us basic tool in proving fixed points results in multiplicative metric spaces.

In 2012, Özavsar and Çevikel [17] gave the multiplicative metric spaces by remarking its topological properties, and introduced concept of multiplicative contraction mapping and proved some fixed point theorems of multiplicative contraction mappings on multiplicative spaces.

It is well known that the set of positive real numbers $\mathbb{R}_{+}$is not complete according to the usual metric. To overcome this problem, in 2008, Bashirov et al. [5] introduced the concept of multiplicative metric spaces as follows:

Definition 1.1. Let $X$ be a nonempty set. A multiplicative metric is a mapping $d: X \times X \rightarrow \mathbb{R}_{+}$satisfying the following conditions:

(i) $d(x, y) \geq 1$ for all $x, y \in X$ and $d(x, y)=1$ if and only if $x=y$;

(ii) $d(x, y)=d(y, x)$ for all $x, y \in X$;

(iii) $d(x, y) \leq d(x, z) \cdot d(z, y)$ for all $x, y, z \in X$ (multiplicative triangle inequality).

Then the mapping $d$ together with $X$, that is, $(X, d)$ is a multiplicative metric space.

Example 1.2. ([17]) Let $\mathbb{R}_{+}^{n}$ be the collection of all $n$-tuples of positive real numbers. Let $d^{*}: \mathbb{R}_{+}^{n} \times \mathbb{R}_{+}^{n} \rightarrow \mathbb{R}$ be defined as follows:

$$
d^{*}(x, y)=\left|\frac{x_{1}}{y_{1}}\right|^{*} \cdot\left|\frac{x_{2}}{y_{2}}\right|^{*} \cdots\left|\frac{x_{n}}{y_{n}}\right|^{*},
$$

where $x=\left(x_{1}, \ldots, x_{n}\right), y=\left(y_{1}, \ldots, y_{n}\right) \in \mathbb{R}_{+}^{n}$ and $|\cdot|^{*}: \mathbb{R}_{+} \rightarrow \mathbb{R}_{+}$is defined 
by

$$
|a|^{*}= \begin{cases}a & \text { if } a \geq 1, \\ \frac{1}{a} & \text { if } a<1 .\end{cases}
$$

Then it is obvious that all conditions of a multiplicative metric are satisfied. Therefore $\left(\mathbb{R}_{+}^{n}, d^{*}\right)$ is a multiplicative metric space.

Example 1.3. ([23]) Let $d: \mathbb{R} \times \mathbb{R} \rightarrow[1, \infty)$ be defined as $d(x, y)=a^{|x-y|}$, where $x, y \in \mathbb{R}$ and $a>1$. Then $d$ is a multiplicative metric and $(\mathbb{R}, d)$ is a multiplicative metric space. We may call it usual multiplicative metric spaces.

Remark 1.4. We note that the Example 1.2 is valid for positive real numbers and Example 1.3 is valid for all real numbers.

Example 1.5. ([23]) Let $(X, d)$ be a metric space. Define a mapping $d_{a}$ on $X$ by

$$
d_{a}(x, y)=a^{d(x, y)}= \begin{cases}1 & \text { if } x=y, \\ a & \text { if } x \neq y,\end{cases}
$$

where $x, y \in X$ and $a>1$. Then $d_{a}$ is a multiplicative metric and $\left(X, d_{a}\right)$ is known as the discrete multiplicative metric space.

Example 1.6. ([1]) Let $X=C^{*}[a, b]$ be the collection of all real-valued multiplicative continuous functions on $[a, b] \subset \mathbb{R}_{+}$. Then $(X, d)$ is a multiplicative metric space with $d$ defined by $d(f, g)=\sup _{x \in[a, b]}\left|\frac{f(x)}{g(x)}\right|$ for arbitrary $f, g \in X$.

Remark 1.7. ([23]) We note that multiplicative metrics and metric spaces are independent.

Indeed, the mapping $d^{*}$ defined in Example 1.2 is multiplicative metric but not metric as it does not satisfy triangular inequality. Consider

$$
d^{*}\left(\frac{1}{3}, \frac{1}{2}\right)+d^{*}\left(\frac{1}{2}, 3\right)=\frac{3}{2}+6=7.5<9=d^{*}\left(\frac{1}{3}, 3\right) .
$$

On the other hand the usual metric on $\mathbb{R}$ is not multiplicative metric as it doesnt satisfy multiplicative triangular inequality, since

$$
d(2,3) \cdot d(3,6)=3<4=d(2,6) .
$$

One can refer to [17] for detailed multiplicative metric topology.

Definition 1.8. Let $(X, d)$ be a multiplicative metric space. Then a sequence $\left\{x_{n}\right\}$ in $X$ is said to be 
(1) a multiplicative convergent to $x$ if for every multiplicative open ball $B_{\epsilon}(x)=\{y \mid d(x, y)<\epsilon\}, \epsilon>1$, there exists $N \in \mathbb{N}$ such that $x_{n} \in B_{\epsilon}(x)$ for all $n \geq N$, that is, $d\left(x_{n}, x\right) \rightarrow 1$ as $n \rightarrow \infty$.

(2) a multiplicative Cauchy sequence if for all $\epsilon>1$, there exists $N \in \mathbb{N}$ such that $d\left(x_{n}, x_{m}\right)<\epsilon$ for all $m, n \geq N$, that is, $d\left(x_{n}, x_{m}\right) \rightarrow 1$ as $n, m \rightarrow \infty$.

(3) We call a multiplicative metric space complete if every multiplicative Cauchy sequence in it is multiplicative convergent to $x \in X$.

Remark 1.9. The set of positive real numbers $\mathbb{R}_{+}$is not complete according to the usual metric. Let $X=\mathbb{R}_{+}$and the sequence $\left\{x_{n}\right\}=\left\{\frac{1}{n}\right\}$. It is obvious $\left\{x_{n}\right\}$ is a Cauchy sequence in $X$ with respect to usual metric and $X$ is not a complete metric space, since $0 \notin \mathbb{R}_{+}$. In case of a multiplicative metric space, we take a sequence $\left\{x_{n}\right\}=\left\{a^{\frac{1}{n}}\right\}$, where $a>1$. Then $\left\{x_{n}\right\}$ is a multiplicative Cauchy sequence since for $n \geq m$,

$$
\begin{aligned}
d\left(x_{n}, x_{m}\right) & =\left|\frac{x_{n}}{x_{m}}\right|=\left|\frac{a^{\frac{1}{n}}}{a^{\frac{1}{m}}}\right|=\left|a^{\frac{1}{n}-\frac{1}{m}}\right| \\
& \leq a^{\frac{1}{m}-\frac{1}{n}}<a^{\frac{1}{m}}<\epsilon \quad \text { if } m>\frac{\log a}{\log \epsilon},
\end{aligned}
$$

where $|a|=\left\{\begin{array}{ll}a & \text { if } a \geq 1, \\ \frac{1}{a} & \text { if } a<1 .\end{array}\right.$ Also, $\left\{x_{n}\right\} \rightarrow 1$ as $n \rightarrow \infty$ and $1 \in \mathbb{R}_{+}$. Hence $(X, d)$ is a complete multiplicative metric space.

In 2012, Özavsar and Çevikel [17] gave the concept of multiplicative contraction mappings and proved some fixed point theorem of such mappings in a multiplicative metric space.

Definition 1.10. Let $f$ be a mapping of a multiplicative metric space $(X, d)$ into itself. Then $f$ is said to be a multiplicative contraction if there exists a real number $\lambda \in[0,1)$ such that

$$
d(f x, f y) \leq d^{\lambda}(x, y) \text { for all } x, y \in X .
$$

Altering distances is introduced by Khan et al. [14] in a metric space. These are control functions which alter the distance between two points in a metric space. Later on various author $([2,3,8,9,22,24,25]$ using altering distances proved common fixed point theorems.

In the similar mode, now we use the altering distances in multiplicative metric spaces. For this we define control functions as follows:

$\Psi=\{\psi \mid \psi:[1, \infty) \rightarrow[1, \infty)$ is continuous and non-decreasing satisfying $\psi(t)=1$ if and only if $t=1\}$. 
$\Phi=\{\phi \mid \phi:[1, \infty) \rightarrow[1, \infty)$ is lower semi-continuous and discontinuous at $t=1, \phi(t)>1$ for all $t \geq 1\}$.

\section{Relations between Various Minimal Commuting Mappings}

In 2015, Kumar et al. [15] introduced the notions of subcompatible and occasionally weakly compatible mappings in a multiplicative metric space.

Definition 2.1. Let $f$ and $g$ be mappings of a multiplicative $(X, d)$ into itself. Then $f$ and $g$ are called

(1) subcompatible if there exists a sequence $\left\{x_{n}\right\}$ in $X$ such that $\lim _{n \rightarrow \infty} f x_{n}=$ $\lim _{n \rightarrow \infty} g x_{n}=t$ for some $t \in X$ and which satisfy $\lim _{n \rightarrow \infty} d\left(f g x_{n}, g f x_{n}\right)=1$;

(2) occasionally weakly compatible if there exists $x \in X$, which is a coincidence point of $f$ and $g$ at which $f$ and $g$ commute, that is, there exists $x \in X$ such that $f x=g x$ implies $f g x=g f x$.

Remark 2.2. Every occasionally weakly compatible mappings are subcompatible. However, the converse is not true, in general.

In 2015, Kang et al. [13] and Jung et al. [11] introduced the notions of compatible and weakly compatible mappings in a multiplicative metric space, respectively.

Definition 2.3. Let $f$ and $g$ be mappings of a multiplicative metric space $(X, d)$ into itself. Then $f$ and $g$ are called

(1) compatible if $\lim _{n \rightarrow \infty} d\left(f g x_{n}, g f x_{n}\right)=1$, whenever $\left\{x_{n}\right\}$ is a sequence in $X$ such that $\lim _{n \rightarrow \infty} f x_{n}=\lim _{n \rightarrow \infty} g x_{n}=t$ for some $t \in X$;

(2) weakly compatible if they commute at coincidence points, that is, if $f t=g t$ for some $t \in X$ implies that $f g t=g f t$.

Remark 2.4. Every compatible mappings are weakly compatible. However, the converse is not true, in general.

In metric spaces, in 2010, Pant and Pant [21], introduced the notion of conditionally commuting mappings. In 2012, Pant and Bisht [20] introduced the notion of conditionally compatible mappings. In 1998, Pant [18] introduced the notion of non-compatible mappings. In 2013, Bisht and Shahzad [7] introduced the notion of faintly compatible mappings.

In similar mode, we introduce the notions in multiplicative metric spaces.

Definition 2.5. Let $f$ and $g$ be mappings of a multiplicative metric space $(X, d)$ into itself. Then $f$ and $g$ are called 
(1) conditionally commuting if the pair commutes on a nonempty subset of the set of coincidence points, whenever the set of coincidences is nonempty;

(2) conditionally compatible if whenever the set of sequences $\left\{y_{n}\right\}$ satisfying $\lim _{n \rightarrow \infty} f y_{n}=\lim _{n \rightarrow \infty} g y_{n}$ is nonempty, there exists a sequence $\left\{z_{n}\right\}$ (distinct from $\left\{y_{n}\right\}$ such that $\lim _{n \rightarrow \infty} f z_{n}=\lim _{n \rightarrow \infty} g z_{n}=t$ and $\lim _{n \rightarrow \infty} d\left(f g z_{n}, g f z_{n}\right)=1$;

(3) non-compatible if the pair is not compatible, that is, there exists a sequence $\left\{x_{n}\right\}$ in $X$ such that $\lim _{n \rightarrow \infty} f x_{n}=\lim _{n \rightarrow \infty} g x_{n}=t$ for some $t \in X$, and $\lim _{n \rightarrow \infty} d\left(f g x_{n}, g f x_{n}\right)$ either $\neq 1$ or non-existent;

(4) faintly compatible if $f$ and $g$ are conditionally compatible and $f$ and $g$ commute on a nonempty subset of coincidence points, whenever the set of coincidences is nonempty.

Clearly every commuting mappings are conditionally commuting. However, the converse is not true, in general.

Example 2.6. Let $X=[0, \infty)$ with a multiplicative metric $d: X \times X \rightarrow$ $[1, \infty)$ be defined by $d(x, y)=a^{|x-y|}$ for all $x, y \in X$ and $a>1$.

Define mappings $f, g: X \rightarrow X$ by

$$
f x=\frac{x+2}{2} \quad \text { and } \quad g x=\frac{x^{2}}{2} \quad \text { for all } x \in X .
$$

Here $f$ and $g$ have a coincidence point $x=2$. Further, $f$ and $g$ are conditionally commuting since they commute at a coincidence point $x=2$.

Also we have

$$
f g x=\frac{x^{2}+4}{4} \text { and } \quad g f x=\frac{x+2^{2}}{8} .
$$

Hence $f$ and $g$ are not commuting.

Every weakly compatible mappings are occasionally weakly compatible. However, the converse is not true, in general.

Example 2.7. Let $X=[0, \infty)$ with multiplicative metric $d: X \times X \rightarrow$ $[1, \infty)$ be defined by $d(x, y)=a^{|x-y|}$ for all $x, y \in X$ and $a>1$.

Define mappings $f, g: X \rightarrow X$ by

$$
f x=3 x \quad \text { and } \quad g x=\frac{x^{2}}{3} \quad \text { for all } x \in X .
$$

Here $f$ and $g$ have two coincidence points $x=0,9$. Further, $f$ and $g$ are ccasionally weakly compatible since they commute at a coincidence point $x=0$. But $f$ and $g$ are not weakly compatible as they are not commuting at $x=9$ as

$$
f g 9=81 \neq 9=g f 9 .
$$


Every occasionally weakly compatible mappings are conditionally compatible. However, the converse is not true, in general.

Example 2.8. Let $X=[0, \infty)$ with a multiplicative metric $d: X \times X \rightarrow$ $[1, \infty)$ be defined by $d(x, y)=a^{|x-y|}$ for all $x, y \in X$ and $a>1$.

Define mappings $f, g: X \rightarrow X$ by

$f x=x^{2} \quad$ for all $x \in X \quad$ and $\quad g x= \begin{cases}x+6 & \text { if } x \in[0,9] \cup(16, \infty), \\ 7 x+18 & \text { if } x \in(9,16]\end{cases}$

Let $\left\{y_{n}\right\}=\left\{3-\frac{1}{n}\right\}$ be a sequence in $X$. Then $\lim _{n \rightarrow \infty} f y_{n}=9$ and $\lim _{n \rightarrow \infty} g y_{n}=$ 9. Hence the set of sequences $\left\{y_{n}\right\}$ satisfying $\lim _{n \rightarrow \infty} f y_{n}=\lim _{n \rightarrow \infty} g y_{n}$ is nonempty.

Now let $\left\{z_{n}\right\}=\left\{3+\frac{1}{n}\right\}$ be a sequence in $X$. Then $\lim _{n \rightarrow \infty} f z_{n}=9$ and $\lim _{n \rightarrow \infty} g z_{n}=9$ and hence

$$
\lim _{n \rightarrow \infty} f g z_{n}=81, \quad \lim _{n \rightarrow \infty} g f z_{n}=81, \quad \lim _{n \rightarrow \infty} d\left(f g z_{n}, g f z_{n}\right)=1 .
$$

So, $f$ and $g$ are conditionally compatible. Here 3 is a coincidence point at which

$$
f g 3=81 \neq 15=g f 3
$$

and hence $f$ and $g$ are not commuting at 3. Thus, $f$ and $g$ are not occasionally weakly compatible.

Every faintly compatible and non-compatible are independent each other.

Example 2.9. Let $\mathrm{X}=[1,5)$ with a multiplicative metric $d: X \times X \rightarrow$ $[1, \infty)$ be defined by $d(x, y)=a^{|x-y|}$ for all $x, y \in X$ and $a>1$.

Define mappings $f, g: X \rightarrow X$ by

$$
f x=\left\{\begin{array}{ll}
5 & \text { if } 1 \leq x<4, \\
1 & \text { if } x \geq 4
\end{array} \text { and } g x= \begin{cases}3 & \text { if } 1 \leq x<4 \\
x-3 & \text { if } x \geq 4\end{cases}\right.
$$

Let $\left\{y_{n}\right\}=\left\{4+\frac{1}{n}\right\}$ be a sequence in $X$. Then $\lim _{n \rightarrow \infty} f y_{n}=1$ and $\lim _{n \rightarrow \infty} g y_{n}=1$ and hence

$$
\lim _{n \rightarrow \infty} f g y_{n}=5, \quad \lim _{n \rightarrow \infty} g f y_{n}=3, \quad \lim _{n \rightarrow \infty} d\left(f g y_{n}, g f y_{n}\right)=a^{2} \neq 1 .
$$

So, $f$ and $g$ are non-compatible. Here 4 is a coincidence point of $f$ and $g$. But

$$
f g 4=6 \neq 3=g f 2 .
$$

Hence $f$ and $g$ are not faintly compatible. 
Example 2.10. Let $X=[0, \infty)$ with a multiplicative metric $d: X \times X \rightarrow$ $[1, \infty)$ be defined by $d(x, y)=a^{|x-y|}$ for all $x, y \in X$ and $a>1$.

Define mappings $f, g: X \rightarrow X$ by

$$
f x=x^{2} \quad \text { for all } x \in X \quad \text { and } \quad g x= \begin{cases}1 & \text { if } x \in(0,2], \\ 0 & \text { if } x \in\{0\} \cup(2, \infty] .\end{cases}
$$

Let $\left\{y_{n}\right\}=\left\{1+\frac{1}{n+1}\right\}$ be a sequence in $X$. Then $\lim _{n \rightarrow \infty} f y_{n}=1$ and $\lim _{n \rightarrow \infty} g y_{n}=1$ and hence

$$
\lim _{n \rightarrow \infty} f g y_{n}=1, \quad \lim _{n \rightarrow \infty} g f y_{n}=1, \quad \lim _{n \rightarrow \infty} d\left(f g y_{n}, g f y_{n}\right)=1 .
$$

So, $f$ and $g$ are not non-compatible.

Now let $\left\{z_{n}\right\}=\{0\}$ be a constant sequence. Then $\lim _{n \rightarrow \infty} f z_{n}=0$ and $\lim _{n \rightarrow \infty} g z_{n}=0$ and hence

$$
\lim _{n \rightarrow \infty} f g z_{n}=0, \quad \lim _{n \rightarrow \infty} g f z_{n}=0, \quad \lim _{n \rightarrow \infty} d\left(f g z_{n}, g f z_{n}\right)=1 .
$$

Further 0 is the only one coincidence point in which $f$ and $g$ commute. So, $f$ and $g$ are faintly compatible.

\section{Main Results}

In 1999, Pant [19] introduced a new notion of continuity, known as reciprocal continuity. In similar mode, we introduced the notions in a multiplicative metric space.

Definition 3.1. [12] Let $f$ and $g$ be mappings of a multiplicative $(X, d)$ into itself. Then $f$ and $g$ are called reciprocally continuous if $\lim _{n \rightarrow \infty} f g x_{n}=f t$ and $\lim _{n \rightarrow \infty} g f x_{n}=g t$, whenever $\left\{x_{n}\right\}$ is a sequence in $X$ such that $\lim _{n \rightarrow \infty} f x_{n}=$ $\lim _{n \rightarrow \infty} g x_{n}=t$ for some $t \in X$.

Now, we prove the following theorem for subcompatible mapping.

Theorem 3.2. Let $A, B, S$ and $T$ be mappings of a complete multiplicative metric space $(X, d)$ into itself satisfying a $(\psi-\phi)$-contractive condition, that is,

$$
\psi(d(A x, B y)) \leq \frac{\psi(M(x, y))}{\phi(N(x, y))}
$$


for all $x, y \in X$ with $x \neq y$, where $\psi \in \Psi, \phi \in \Phi$,

$$
\begin{gathered}
M(x, y)=\max \left\{d(S x, T y),(d(S x, A x) \cdot d(T y, B y))^{1 / 2},\right. \\
\left.(d(S x, B y) \cdot d(T y, A x))^{1 / 2}\right\}
\end{gathered}
$$

and

$$
N(x, y)=\min \{d(S x, T y), d(S x, A x), d(T y, B y)\}
$$

$\left(C_{2}\right)$ the pairs $A, S$ and $B, T$ are reciprocally continuous.

Assume that the pairs $A, S$ and $B, T$ are subcompatible. Then $A, B, S$ and $T$ have a unique common fixed point in $X$.

Proof. Since the pairs $A, S$ and $B, T$ are subcompatible and reciprocally continuous, there exists sequences $\left\{x_{n}\right\}$ and $\left\{y_{n}\right\}$ in $X$ such that $\lim _{n \rightarrow \infty} A x_{n}=$ $\lim _{n \rightarrow \infty} S x_{n}=w$ for some $w \in X$ and which satisfy $\lim _{n \rightarrow \infty} d\left(A S x_{n}, S A x_{n}\right)=$ $d(A w, S w)=1$ and $\lim _{n \rightarrow \infty} B y_{n}=\lim _{n \rightarrow \infty} T y_{n}=z$ for some $z \in X$ and which satisfy $\lim _{n \rightarrow \infty} d\left(B T y_{n}, T B y_{n}\right)=d(B z, T z)=1$.

Therefore, $A w=S w$ and $B z=T z$, that is $w$ is a coincidence point of $A$ and $S$, and $z$ is a coincidence point of $B$ and $T$.

Now we claim that $w=z$. Putting $x=x_{n}$ and $y=y_{n}$ in $\left(C_{1}\right)$, we get

$$
\psi\left(d\left(A x_{n}, B y_{n}\right)\right) \leq \frac{\psi\left(M\left(x_{n}, y_{n}\right)\right)}{\phi\left(N\left(x_{n}, y_{n}\right)\right)},
$$

where

$$
\begin{gathered}
M\left(x_{n}, y_{n}\right)=\max \left\{d\left(S x_{n}, T y_{n}\right),\left(d\left(S x_{n}, A x_{2 n}\right) \cdot d\left(T y_{n}, B y_{n}\right)\right)^{1 / 2}\right. \\
\left.\left(d\left(S x_{n}, B y_{n}\right) \cdot d\left(T y_{n}, A x_{n}\right)\right)^{1 / 2}\right\}
\end{gathered}
$$

and

$$
N\left(x_{n}, y_{n}\right)=\min \left\{d\left(S x_{n}, T y_{n}\right), d\left(S x_{n}, A x_{2 n}\right), d\left(T y_{n}, B y_{n}\right)\right\}
$$

Letting $n \rightarrow \infty$, we have

$$
\lim _{n \rightarrow \infty} M\left(x_{n}, y_{n}\right)=d(w, z)
$$

and

$$
\lim _{n \rightarrow \infty} N\left(x_{n}, y_{n}\right)=1 .
$$

Since $\phi$ is discontinuous at $t=1$. Therefore, we have

$$
\psi(d(t, z)) \leq \frac{\psi(w, z)}{\lim _{n \rightarrow \infty} \phi\left(N\left(x_{2 n}, y_{n}\right)\right)}<\psi(w, z),
$$


which is a contradiction. Hence $w=z$.

Next we show that $A z=z$. Putting $x=z$ and $y=y_{n}$ in $\left(C_{1}\right)$, we have

$$
\psi\left(d\left(A z, B y_{n}\right)\right) \leq \frac{\psi\left(M\left(z, y_{n}\right)\right)}{\phi\left(N\left(z, y_{n}\right)\right)},
$$

where

$$
\begin{gathered}
M\left(z, y_{n}\right)=\max \left\{d\left(S z, T y_{n}\right),\left(d(S z, A z) \cdot d\left(T y_{n}, B y_{n}\right)\right)^{1 / 2}\right. \\
\left.\left(d\left(S z, B y_{n}\right) \cdot d\left(T y_{n}, A z\right)\right)^{1 / 2}\right\}
\end{gathered}
$$

and

$$
N\left(z, y_{n}\right)=\min \left\{d\left(S z, T y_{n}\right), d(S z, A z), d\left(T y_{n}, B y_{n}\right)\right\}
$$

Letting $n \rightarrow \infty$, we get

$$
\lim _{n \rightarrow \infty} M\left(z, y_{n}\right)=d(z, A z)
$$

and

$$
\lim _{n \rightarrow \infty} N\left(z, y_{n}\right)=1
$$

Since $\phi$ is discontinuous at $t=1$. Therefore, we have

$$
\psi(d(A z, z)) \leq \frac{\psi(z, A z)}{\lim _{n \rightarrow \infty} \phi\left(N\left(z, y_{n}\right)\right)}<\psi(z, A z)
$$

which implies that $A z=z$.

Finally we claim that $B z=z$. Putting $x=z$ and $y=z$ in $\left(C_{1}\right)$, we have

$$
\psi(d(A z, B z)) \leq \frac{\psi(M(z, z))}{\phi(N(z, z))},
$$

where

$$
\begin{gathered}
M(z, z)=\max \left\{d(S z, T z),(d(S z, A z) \cdot d(T z, B z))^{1 / 2},\right. \\
\left.(d(S z, B z) \cdot d(T z, A z))^{1 / 2}\right\}
\end{gathered}
$$

and

$$
N(z, z)=\min \{d(S z, T z), d(S z, A z), d(T z, B z)\}
$$

Hence

$$
M(z, z)=d(z, B z) \quad \text { and } \quad N(z, z)=1
$$


Further we have

$$
\begin{aligned}
\psi(d(z, B z)) & =\psi(d(A z, B z)) \\
& \leq \frac{\psi(d(z, B z))}{\phi(N(z, z))}<\psi(d(z, B z)),
\end{aligned}
$$

which is a contradiction since $\phi(t)>1$ for $t \geq 1$. Hence we have $B z=z$. Hence $B z=A z=S z=T z=z$, that is $z$ is a common fixed point of $A, B, S$ and $T$.

Uniqueness follows easily. Therefore $A, B, S$ and $T$ have a unique common fixed point. This completes the proof.

Next, we prove the following theorem for occasionally weakly compatible mappings without reciprocal continuity

Theorem 3.3. Let $A, B, S$ and $T$ be mappings of a complete multiplicative metric space $(X, d)$ into itself satisfying the condition $\left(C_{1}\right)$.

Assume that the pairs $A, S$ and $B, T$ are occasionally weakly compatible. Then $A, B, S$ and $T$ have a unique common fixed point in $X$.

Proof. Since the pair $A, S$ is occasionally weakly compatible, there exists $u \in X$ such that $A u=S u=z$ (say) and $A S u=S A u$, that is, $A z=S z=z^{\prime}$ (say). Since the pair $B, T$ is occasionally weakly compatible, there exists $v \in X$ such that $B v=T v=w$ (say) and $B T v=T B v=w^{\prime}$ (say).

Now, we claim that $z^{\prime}=w^{\prime}$. Putting $x=z$ and $y=w$ in $\left(C_{1}\right)$, we have

$$
\psi(d(A z, B w)) \leq \frac{\psi(M(z, w))}{\phi(N(z, w))},
$$

where

$$
\begin{aligned}
M(z, w)= & \max \left\{d(S z, T w),(d(S z, A z) \cdot d(T w, B w))^{1 / 2},\right. \\
& \left.(d(S z, B w) \cdot d(T w, A z))^{1 / 2}\right\} \\
= & d\left(z^{\prime}, w^{\prime}\right)
\end{aligned}
$$

and

$$
\begin{aligned}
N(u, w) & =\min \{d(S u, T w), d(S u, A u), d(T w, B w)\} \\
& =1 .
\end{aligned}
$$

Hence we obtain

$$
\psi\left(d\left(z^{\prime}, w^{\prime}\right)\right) \leq \frac{\psi\left(d\left(z^{\prime}, w^{\prime}\right)\right.}{\phi(N(z, w))}<\psi\left(d\left(z^{\prime}, w^{\prime}\right),\right.
$$

which is a contradiction since $\phi(t)>1$ for $t \geq 1$. Thus $z^{\prime}=w^{\prime}$. Hence we have

$$
A z=S z=z^{\prime}=w^{\prime}=B w=T w .
$$


Next we prove that $z=w^{\prime}$. Putting $x=u$ and $y=w$ in $\left(C_{1}\right)$, we have

$$
\psi(d(A u, B w)) \leq \frac{\psi(M(u, w))}{\phi(N(u, w))},
$$

where

$$
\begin{aligned}
M(u, w)= & \max \left\{d(S u, T w),(d(S u, A u) \cdot d(T w, B w))^{1 / 2},\right. \\
& \left.(d(S u, B w) \cdot d(T w, A u))^{1 / 2}\right\} \\
= & d\left(z, w^{\prime}\right)
\end{aligned}
$$

and

$$
\begin{aligned}
N(z, w) & =\min \{d(S z, T w), d(S z, A z), d(T w, B w)\} \\
& =1
\end{aligned}
$$

Hence we obtain

$$
\psi\left(d\left(z, w^{\prime}\right)\right) \leq \frac{\psi\left(d\left(z, w^{\prime}\right)\right)}{\phi(N(z, w))}<\psi\left(d\left(z, w^{\prime}\right)\right),
$$

which is a contradiction. Thus $z=w^{\prime}$. Hence we have $A z=S z=z$ and $B w=T w=z$.

Again, we claim that $z=w$. Putting $x=z$ and $y=v$ in $\left(C_{1}\right)$, we have

$$
\psi(d(A z, B v)) \leq \frac{\psi(M(z, v))}{\phi(N(z, v))}
$$

where

$$
\begin{aligned}
M(z, v)= & \max \left\{d(S z, T v),(d(S z, A z) \cdot d(T v, B v))^{1 / 2}\right. \\
& \left.(d(S z, B v) \cdot d(T v, A z))^{1 / 2}\right\} \\
= & d(z, w)
\end{aligned}
$$

and

$$
\begin{aligned}
N(z, v) & =\min \{d(S z, T v), d(S z, A z), d(T v, B V)\} \\
& =1 .
\end{aligned}
$$

Hence we obtain

$$
\psi(d(z, w)) \leq \frac{\psi(d(z, w))}{\phi(N(z, v))}<\psi(d(z, w)),
$$

which is a contradiction. So we have $z=w$. Thus we have $A z=S z=B z=$ $T z=z$. Hence $z$ is a common fixed point of $A, B, S$ and $T$.

Uniqueness follows easily. Therefore $A, B, S$ and $T$ have a unique common fixed point. This completes the proof. 
Next, we prove the following theorem for conditionally commuting.

Theorem 3.4. Let $A, B, S$ and $T$ be mappings of a complete multiplicative metric space $(X, d)$ into itself satisfying the condition $\left(C_{1}\right)$. Suppos that

$$
A(X) \subset T(X) \text { and } B(X) \subset S(X)
$$

$\left(C_{4}\right)$ One of the subspace $A X$ or BX or SX or TX be closed.

Assume that the pairs $A, S$ and $B, T$ are conditionally commuting. Then $A, B, S$ and $T$ have a unique common fixed point in $X$.

Proof. Let $x_{0} \in X$ be an arbitrary point. Since $A(X) \subset T(X)$ and $B(X) \subset$ $S(X)$, there exist a point $x_{1} \in X$ such that $A x_{0}=T x_{1}$ and for this point $x_{1} \in X$, there exists a point $x_{2} \in X$ such that $B x_{1}=S x_{2}$. Continuing this process, we can define a sequence $\left\{y_{n}\right\}$ in $X$ such that

$$
y_{2 n+1}=A x_{2 n}=T x_{2 n+1}, \quad y_{2 n+2}=B x_{2 n+1}=S x_{2 n+2} \quad \text { for } n \geq 0 .
$$

From the proof of [16, Theorem 3.1], $\left\{y_{n}\right\}$ is a multiplicative metric space. Since $(X, d)$ is complete, there exists $z \in X$ such that

$$
A x_{2 n} \rightarrow z, \quad T x_{2 n+1} \rightarrow z, \quad B x_{2 n+1} \rightarrow z, \quad S x_{2 n+2} \rightarrow z .
$$

Assume that $S X$ is closed, there exists $v \in X$ such that $S v=z$.

Now we claim that $A v=z$. Putting $x=v$ and $y=x_{2 n+1}$ in $\left(C_{1}\right)$, we have

$$
\psi\left(d\left(A v, B x_{2 n+1}\right)\right) \leq \frac{\psi\left(M\left(v, x_{2 n+1}\right)\right.}{\phi\left(N\left(v, x_{2 n+1}\right)\right)},
$$

where

$$
\begin{gathered}
M\left(v, x_{2 n+1}\right)=\max \left\{d\left(S v, T x_{2 n+1}\right),\left(d(S v, A v) \cdot d\left(T x_{2 n+1}, B x_{2 n+1}\right)\right)^{1 / 2}\right. \\
\left.\left(d\left(S v, B x_{2 n+1}\right) \cdot d\left(T x_{2 n+1}, A v\right)\right)^{1 / 2}\right\}
\end{gathered}
$$

and

$$
N\left(v, x_{2 n+1}\right)=\min \left\{d\left(S v, T x_{2 n+1}\right), d(S v, A v), d\left(T x_{2 n+1}, B x_{2 n+1}\right)\right\} .
$$

Letting $n \rightarrow \infty$, we have

$$
\begin{aligned}
\lim _{n \rightarrow \infty} M\left(v, x_{2 n+1}\right)= & \max \left\{d(S v, z),(d(S v, A v) \cdot d(z, z))^{1 / 2}\right. \\
& \left.(d(S v, z) \cdot d(z, A v))^{1 / 2}\right\} \\
= & d^{1 / 2}(z, A v)
\end{aligned}
$$


and

$$
\begin{aligned}
\lim _{n \rightarrow \infty} N\left(v, x_{2 n+1}\right) & =\max \{d(S v, z), d(S v, A v), d(z, z)\} \\
& =1 .
\end{aligned}
$$

Hence we get

$$
\psi(d(A v, z)) \leq \frac{\psi\left(d^{1 / 2}(z, A v)\right)}{\lim _{n \rightarrow \infty} \phi\left(N\left(v, x_{2 n+1}\right)\right)}<\psi\left(d^{1 / 2}(z, A v)\right),
$$

which is a contradiction since $\phi$ is discontinuous at $t=1$. Thus we obtain $A v=z$. Since $A X \subset T X$, there exists $u \in X$ such that $A v=T u=z$.

Next, we claim that $B u=z$. Putting $x=x_{2 n}$ and $y=u$ in $\left(C_{1}\right)$, we get

$$
\psi\left(d\left(A x_{2 n}, B u\right)\right) \leq \frac{\psi\left(M\left(x_{2 n}, u\right)\right)}{\phi\left(N\left(x_{2 n}, u\right)\right)},
$$

where

$$
\begin{gathered}
M\left(x_{2 n}, u\right)=\max \left\{d\left(S x_{2 n}, T u\right),\left(d\left(S x_{2 n}, A x_{2 n}\right) \cdot d(T u, B u)\right)^{1 / 2},\right. \\
\left.\left(d\left(S x_{2 n}, B u\right) \cdot d\left(T u, A x_{2 n}\right)\right)^{1 / 2}\right\}
\end{gathered}
$$

and

$$
N\left(x_{2 n}, u\right)=\min \left\{d\left(S x_{2 n}, T u\right), d\left(S x_{2 n}, A x_{2 n}\right), d(T u, B u)\right\} .
$$

Letting $n \rightarrow \infty$, we have

$$
\begin{aligned}
\lim _{n \rightarrow \infty} M\left(x_{2 n}, u\right)= & \max \left\{d(z, T u),(d(z, z) \cdot d(T u, B u))^{1 / 2},\right. \\
& \left.(d(z, B u) \cdot d(T u, z))^{1 / 2}\right\} \\
= & d^{1 / 2}(z, B u)
\end{aligned}
$$

and

$$
\begin{aligned}
\lim _{n \rightarrow \infty} N\left(x_{2 n}, u\right) & =\min \{d(z, T u), d(z, z), d(T u, B u)\} \\
& =1 .
\end{aligned}
$$

Hence we get

$$
\psi(d(z, B u)) \leq \frac{\psi\left(d^{1 / 2}(z, B u)\right)}{\lim _{n \rightarrow \infty} \phi\left(N\left(x_{2 n}, u\right)\right)}<\psi\left(d^{1 / 2}(z, B u)\right)
$$

which is a contradiction since $\phi$ is discontinuous at $t=1$. So we have $B u=z$. Thus we have $A v=S v=T u=B u=z$. Hence we conclude that $v$ is a coincidence point of $A$ and $S$ and $u$ is a coincidence point of $B$ and $T$. 
Case 1. $A$ and $S$ commutes at $v$ and $B$ and $T$ commutes at $u$.

Hence $A S v=S A v$, that is, $A z=S z$ and $B T u=T B u$, that is, $B z=T z$.

Now, we claim that $A z=z$. Putting $x=z$ and $y=x_{2 n+1}$ in $\left(C_{1}\right)$, we have

$$
\psi\left(d\left(A z, B x_{2 n+1}\right)\right) \leq \frac{\psi\left(M\left(z, x_{2 n+1}\right)\right.}{\phi\left(N\left(z, x_{2 n+1}\right)\right)},
$$

where

$$
\begin{gathered}
M\left(z, x_{2 n+1}\right)=\max \left\{d\left(S z, T x_{2 n+1}\right),\left(d(S z, A z) \cdot d\left(T x_{2 n+1}, B x_{2 n+1}\right)\right)^{1 / 2},\right. \\
\left.\left(d\left(S z, B x_{2 n+1}\right) \cdot d\left(T x_{2 n+1}, A z\right)\right)^{1 / 2}\right\} .
\end{gathered}
$$

and

$$
N\left(z, x_{2 n+1}\right)=\min \left\{d\left(S z, T x_{2 n+1}\right), d(S z, A z), d\left(T x_{2 n+1}, B x_{2 n+1}\right)\right\} .
$$

Letting $n \rightarrow \infty$, we have

$$
\begin{aligned}
\lim _{n \rightarrow \infty} M\left(z, x_{2 n+1}\right)= & \max \left\{d(S z, z),(d(S z, A z) \cdot d(z, z))^{1 / 2}\right. \\
& \left.(d(S z, z) \cdot d(z, A z))^{1 / 2}\right\} \\
= & d(z, A z)
\end{aligned}
$$

and

$$
\begin{aligned}
\lim _{n \rightarrow \infty} N\left(z, x_{2 n+1}\right) & =\max \{d(S z, z), d(S z, A z), d(z, z)\} \\
& =1
\end{aligned}
$$

Hence we get

$$
\psi(d(A z, z)) \leq \frac{\psi(d(z, A z))}{\lim _{n \rightarrow \infty} \phi\left(N\left(z, x_{2 n+1}\right)\right)}<\psi(d(z, A z)),
$$

which is a contradiction since $\phi$ is discontinuous at $t=1$. So we have $A z=z$.

Next we show that $A z=B z$. Putting $x=z$ and $y=z$ in $\left(C_{1}\right)$, we have

$$
\psi(d(A z, B z)) \leq \frac{\psi(M(z, z))}{\phi(N(z, z))},
$$

where

$$
\begin{aligned}
M(z, z)= & \max \left\{d(S z, T z),(d(S z, A z) \cdot d(T z, B z))^{1 / 2}\right. \\
& \left.(d(S z, B z) \cdot d(T z, A z))^{1 / 2}\right\} \\
= & d(A z, B z)
\end{aligned}
$$


and

$$
\begin{aligned}
N(z, z) & =\min \{d(S z, T z), d(S z, A z), d(T z, B z)\} \\
& =1 .
\end{aligned}
$$

Hence we have

$$
\psi(d(A z, B z)) \leq \frac{\psi(d(A z, B z))}{\phi(N(z, z))}<\psi(d(A z, B z)),
$$

which is a contradiction since $\phi(t)>1$ for $t \geq 1$. Thus, we have $B z=T z=$ $A z=S z=z$, that is, $z$ is a common fixed point of $A, B, S$ and $T$.

Case 2. $A$ and $S$ commute at $v$, but $B$ and $T$ do not commute at $u$.

Since $A$ and $S$ commute, $A S v=S A v=z^{\prime}$ (say), that is, $A z=S z=z^{\prime}$. Since $A v=S v=z$. by $B$ and $T$ is conditionally commuting, there exists $r \in X$ such that $B r=T r=w$ (say) and also $B T r=T B r=w^{\prime}$ (say), that is, $B w=T w=w^{\prime}$.

Now we shall prove that $z^{\prime}=w^{\prime}$. Putting $x=z$ and $y=w$ in $\left(C_{1}\right)$, we have

$$
\psi(d(A z, B w)) \leq \frac{\psi(M(z, w))}{\phi(N(z, w))},
$$

where

$$
\begin{aligned}
M(z, w)= & \max \left\{d(S z, T w),(d(S z, A z) \cdot d(T w, B w))^{1 / 2},\right. \\
& \left.(d(S z, B w) \cdot d(T w, A z))^{1 / 2}\right\} \\
= & d\left(z^{\prime}, w^{\prime}\right)
\end{aligned}
$$

and

$$
\begin{aligned}
N(z, w) & =\min \{d(S z, T w), d(S z, A z), d(T w, B w)\} \\
& =1 .
\end{aligned}
$$

Hence we obtain

$$
\psi\left(d\left(z^{\prime}, w^{\prime}\right)\right) \leq \frac{\psi\left(d\left(z^{\prime}, w^{\prime}\right)\right)}{\phi(N(z, w))}<\psi\left(d\left(z^{\prime}, w^{\prime}\right)\right),
$$

which is a contradiction since $\phi(t)>1$ for $t \geq 1$. Hence $z^{\prime}=w^{\prime}$. Thus, we have $A z=S z=z^{\prime}=B w=T w$.

Now we claim that $z=z^{\prime}$. Putting $x=v$ and $y=w$ in $\left(C_{1}\right)$, we have

$$
\psi(d(A v, B w)) \leq \frac{\psi(M(v, w))}{\phi(N(v, w))},
$$

where

$$
\begin{aligned}
M(v, w)= & \max \left\{d(S v, T w),(d(S v, A v) \cdot d(T w, B w))^{1 / 2},\right. \\
& \left.(d(S v, B w) \cdot d(T w, A v))^{1 / 2}\right\} \\
= & d\left(z, z^{\prime}\right)
\end{aligned}
$$


and

$$
\begin{aligned}
N(v, w) & =\min \{d(S v, T w), d(S v, A v), d(T w, B w)\} \\
& =1
\end{aligned}
$$

Hence, we obtain

$$
\psi\left(d\left(z, z^{\prime}\right)\right) \leq \frac{\psi\left(d\left(z, z^{\prime}\right)\right)}{\phi(N(z, w))}<\psi\left(d\left(z, z^{\prime}\right)\right)
$$

which is a contradiction Thus $z=z^{\prime}$. Hence $A z=S z=z=B w=T w$.

Next we claim that $z=w$. Putting $x=z$ and $y=r$ in $\left(C_{1}\right)$, we have

$$
\psi(d(A z, B r)) \leq \frac{\psi(M(z, r))}{\phi(N(z, r))},
$$

where

$$
\begin{aligned}
M(z, r)= & \max \left\{d(S z, T r),(d(S z, A z) \cdot d(\operatorname{Tr}, B r))^{1 / 2}\right. \\
& \left.(d(S z, B r) \cdot d(\operatorname{Tr}, A z))^{1 / 2}\right\} \\
= & d(z, w)
\end{aligned}
$$

and

$$
\begin{aligned}
N(z, r) & =\min \{d(S z, T r), d(S z, A z), d(T r, B r)\} \\
& =1 .
\end{aligned}
$$

Hence we obtain

$$
\psi(d(z, w)) \leq \frac{\psi(d(z, w)}{\phi(N(z, r))}<\psi(d(z, w)),
$$

which is a contradiction. So we have $z=w$. Thus $A z=S z=z=B z=T z$, that is, $z$ is a common fixed point of $A, B, S$ and $T$.

Case 3. $B$ and $T$ commute at $u$, but $A$ and $S$ do not commute at $v$.

This case is similar to Case 2 .

Case 4 . $A$ and $S$ do not commute at $v$, but $B$ and $T$ do not commute at $u$.

Since $A$ and $S$ be conditionally commuting, there exists $p \in X$ such that $A p=S p=p^{\prime}$ (say) and $A S p=S A p$, that is, $A p^{\prime}=S p^{\prime}$. Also $B$ and $T$ is conditionally commuting, there exists $q \in X$ such that $B q=T q=q$ (say) and $B T q=T B q$, that is, $B q^{\prime}=T q^{\prime}$. The rest proof is similar to the proof of Case 2.

Similarly, we can also complete the proof when $A X$ or $B X$ or $T X$ is closed.

Uniqueness follows easily. Therefore $A, B, S$ and $T$ have a unique common fixed point. This completes the proof. 
Next we prove the following theorem for conditionally compatible and noncompatible.

Theorem 3.5. Let $A, B, S$ and $T$ be mappings of a complete multiplicative metric space $(X, d)$ into itself satisfying the conditions $\left(C_{1}\right)$ and $\left(C_{2}\right)$.

Assume that the pairs $A, S$ and $B, T$ are conditionally compatible and noncompatible. Then $A, B, S$ and $T$ have a unique common fixed point in $X$.

Proof. Since $A$ and $S$ is non-compatible, there exists a sequence $\left\{x_{n}\right\}$ in $X$ such that $\lim _{n \rightarrow \infty} A x_{n}=\lim _{n \rightarrow \infty} S x_{n}=t_{1}$ for some $t_{1} \in X$ and $\lim _{n \rightarrow \infty} d\left(A S x_{n}, S A x_{n}\right) \neq$ 1. Also since $B$ and $T$ is non-compatible, there exists a sequence $\left\{y_{n}\right\}$ in $X$ such that $\lim _{n \rightarrow \infty} B y_{n}=\lim _{n \rightarrow \infty} T y_{n}=t_{2}$ for some $t_{2} \in X$ and $\lim _{n \rightarrow \infty} d\left(B T y_{n}, T B y_{n}\right) \neq 1$. Since the paies $A, S$ and $B, T$ are conditionally compatible, there exists $\left\{z_{n}\right\}$ and $\left\{v_{n}\right\}$ in $X$ such that $\lim _{n \rightarrow \infty} A z_{n}=\lim _{n \rightarrow \infty} S z_{n}=z$ for some $z \in X$ and $\lim _{n \rightarrow \infty} d\left(A S z_{n}, S A z_{n}\right)=1$. and $\lim _{n \rightarrow \infty} B v_{n}=\lim _{n \rightarrow \infty} T v_{n}=v$ for some $v \in X$ and $\lim _{n \rightarrow \infty} d\left(B T v_{n}, T B v_{n}\right)=1$.

Also since the pairs $A, S$ and $B, T$ are reciprocally continuous, $\lim _{n \rightarrow \infty} A S z_{n}=$ $A z$ and $\lim _{n \rightarrow \infty} S A z_{n}=S z$ and hence $A z=S z$. Similarly $\lim _{n \rightarrow \infty} B T v_{n}=B v$ and $\lim _{n \rightarrow \infty} T B v_{n}=T v$ and hence $B v=T v$.

Now we claim that $z=v$. Putting $x=z_{n}$ and $y=v_{n}$ in $\left(C_{1}\right)$, we have

$$
\psi\left(d\left(A z_{n}, B v_{n}\right)\right) \leq \frac{\psi\left(M\left(z_{n}, v_{n}\right)\right)}{\phi\left(N\left(z_{n}, v_{n}\right)\right)}
$$

where

$$
\begin{gathered}
M\left(z_{n}, v_{n}\right)=\max \left\{d\left(S z_{n}, T v_{n}\right),\left(d\left(S z_{n}, A z_{n}\right) \cdot d\left(T v_{n}, B v_{n}\right)\right)^{1 / 2}\right. \\
\left.\left(d\left(S z_{n}, B v_{n}\right) \cdot d\left(T v_{n}, A z_{n}\right)\right)^{1 / 2}\right\}
\end{gathered}
$$

and

$$
N\left(z_{n}, v_{n}\right)=\min \left\{d\left(S z_{n}, T v_{n}\right), d\left(S z_{n}, A z_{n}\right), d\left(T v_{n}, B v_{n}\right)\right\}
$$

Letting $n \rightarrow \infty$, we have

$$
\begin{aligned}
\lim _{n \rightarrow \infty} M\left(z_{n}, v_{n}\right) & =\max \left\{d(z, v), 1,(d(z, v) \cdot d(v, z))^{1 / 2}\right. \\
& =d(u, v)
\end{aligned}
$$

and

$$
\left.\lim _{n \rightarrow \infty} N\left(z_{n}, v_{n}\right)\right)=1 .
$$


Since $\phi$ is discontinuous at $t=1$, we obtain

$$
\psi\left(d(z, v) \leq \frac{\psi(d(z, v))}{\lim _{n \rightarrow \infty} \phi\left(N\left(z_{n}, v_{n}\right)\right)}<\psi(d(z, v))\right.
$$

which is a contradiction. Hence $z=v$.

Next, we show that $A z=z$. Putting $x=z$ and $y=v_{n}$ in $\left(C_{1}\right)$, we have

$$
\psi\left(d\left(A z, B v_{n}\right)\right) \leq \frac{\psi\left(M\left(z, v_{n}\right)\right)}{\phi\left(N\left(z, v_{n}\right)\right)},
$$

where

$$
\begin{gathered}
M\left(z, v_{n}\right)=\max \left\{d\left(S z, T v_{n}\right),\left(d(S z, A z) \cdot d\left(T v_{n}, B v_{n}\right)\right)^{1 / 2}\right. \\
\left.\left(d\left(S z, B v_{n}\right) \cdot d\left(T v_{n}, A z\right)\right)^{1 / 2}\right\}
\end{gathered}
$$

and

$$
N\left(z, v_{n}\right)=\min \left\{d\left(S z, T v_{n}\right), d(S z, A z), d\left(T v_{n}, B v_{n}\right)\right\}
$$

Letting $n \rightarrow \infty$, we have

$$
\begin{aligned}
\lim _{n \rightarrow \infty} M\left(z, v_{n}\right) & =\max \left\{d(A z, z), 1,(d(A z, z) \cdot d(v, A z))^{1 / 2}\right\} \\
& =d(A z, z)
\end{aligned}
$$

and

$$
\lim _{n \rightarrow \infty} N\left(z, v_{n}\right)=\min \{d(A z, z, 1,1\}=1
$$

Since $\phi$ is discontinuous at $t=1$, we obtain

$$
\psi\left(d(A z, z) \leq \frac{\psi(d(A z, z))}{\lim _{n \rightarrow \infty} \phi\left(N\left(z, v_{n}\right)\right)}<\psi(d(A z, z)),\right.
$$

which is a contradiction. Hence $A z=z$.

Similarly putting $x=z_{n}$ and $y=z$ in $\left(C_{1}\right)$, we obtain $B z=z$. Thus we have $A z=B z=S z=T z=z$. Hence $u$ is a common fixed point of $A, B, S$ and $T$.

Uniqueness follows easily. Therefore $A, B, S$ and $T$ have a unique common fixed point. This completes the proof.

Next we prove the following theorem for faintly compatible and non-compatible. 
Theorem 3.6. Let $A, B, S$ and $T$ be mappings of a complete multiplicative metric space $(X, d)$ into itself satisfying the conditions $\left(C_{1}\right)$ and $\left(C_{2}\right)$.

Assume that the pairs $A, S$ and $B, T$ are faintly compatible and non-compatible. Then $A, B, S$ and $T$ have a unique common fixed point in $X$.

Proof. Since the pairs $A, S$ and $B, T$ are faintly compatible, by definition of faintly compatible, the pairs $A, S$ and $B, T$ are also conditionally compatible. The result follows immediately from Theorem 3.5.

Acknowledgment. This work was supported by the Dong-A University research fund.

\section{References}

[1] M. Abbas, B. Ali, Y.I. Suleiman, Common fixed points of locally contractive mappings in multiplicative metric spaces with application, Int. J. Math. Math. Sci., 2015 (2015), Article ID 218683, 7 pages. doi: 10.1155/2015/218683.

[2] M. Abbas, D. Đorić, Common fixed point theorem for four mappings satisfying generalized weak contractive condition, Filomat 24 (2010), 1-10. doi: 10.2298/FIL1002001A

[3] M. Abbas, M.A. Khan, Common fixed point theorem of two mappings satisfying a generalized weak contractive condition, Int. J. Math. Math. Sci., 2009 (2009), Article ID 131068, 9 pages. doi: 10.1155/2009/131068

[4] S. Banach, Sur les opérations dans les ensembles abstracts et leur application aux équations intégrales, Fund. Math., 3 (1922), 133-181.

[5] A.E. Bashirov, E.M. Kurplnara, A. Ozyapici, Multiplicative calculus and its applicatiopns, J. Math. Anal. Appl., 337 (2008), 36-48. doi: 10.1016/j.jmaa.2007.03.081

[6] A.E. Bashirov, E. Msrl, Y. Tandoğdu, A. Özyapc, On modeling with multiplicative differential equations, Appl. Math. J. Chinese Univ. Ser. B, 26 (2011), 425-438. doi: 10.1007/s11766-011-2767-6

[7] R. K. Bisht, N. Shahzad, Faintly compatible mappings and common fied points, Fixed Point Theory Appl., 156 (2013), 9 pages

[8] P.N. Dutta, B.S. Choudhury, A generalization of contraction principle in metric spaces, Fixed Point Theory Appl., (2008) (2008), Article ID 406368, 8 pages. doi: $10.1155 / 2008 / 406368$

[9] D. Đorić, Common fixed point for generalized $(\psi, \phi)$-weak contractions, Appl. Math. Lett., 22 (2009), 1896-1900. doi: 10.1016/j.aml.2009.08.001

[10] L. Florack, H. V. Assen, Multiplicative calculus in biomedical image analysis, J. Math. Imaging Vision, 42 (2012), 64-75. doi: 10.1007/s10851-011-0275-1

[11] C.Y. Jung, P. Kumar, S. Kumar, S.M. Kang, Common fixed points for weakly compatible mappings satisfying implicit functions in multiplicative metric spaces, Int. J. Pure Appl. Math., 102 (2015), 547-561. doi: 10.12732/ijpam.v102i3.12 
[12] S.M. Kang, P. Kumar, S. Kumar, Common fixed points for compatible mappings of types in multiplicative metric spaces, Int. J. Math. Anal., 9 (2015), 1755-1767. doi: 10.12988/ijma.2015.53104

[13] S.M. Kang, P. Kumar, S. Kumar, P. Nagpal, S.K Garg, Common fixed points for compatible mappings and its variants in multiplicative metric spaces, Int. J. Pure Appl. Math., 102 (2015), 383-406. doi: 10.12732/ijpam.v102i2.14

[14] M.S. Khan, M. Swaleh, S. Sessa, Fixed point theorems by altering distances between the points, Bull. Austral. Math. Soc., 30 (1984), 1-9.

[15] P. Kumar, S. Kumar and S.M. Kang, Common fixed point theorems for subcompatible and occasionally weakly compatible mappings in multiplicative metric spaces, Int. J. Math. Anal., 9 (2015), 1785-1794. doi: 10.12988/ijma.2015.53113

[16] Y.C. Kwun, P. Nagpal, S. Kumar, S.K. Garg, S.M. Kang, Fixed points theorems for compatible mappings and its variants satisfying $(\psi-\phi)$-contractive conditions, Int. J. Pure Appl. Math., (accepted).

[17] M. Özavsar, A.C. Çevikel, Fixed points of multiplicative contraction mappings on multiplicative metric spaces, ArXiv:1205.5131v1 [math.GM] (2012), 14 pages.

[18] R.P. Pant, Common fixed point theorems for contractive maps, J. Math. Anal. Appl., 226 (1998), 251-258. doi: 10.1006/jmaa.1998.6029

[19] R.P. Pant, A common fixed point theorem under a new condition, Indian J. Pure Appl. Math., 30 (1999), 147-152.

[20] R.P. Pant, R.K. Bisht, Occasionally weakly compatible mappings and fixed points, Bull. Belg. Math. Soc. Simon Stevin, 19 (2012), 655-661.

[21] V. Pant, R.P. Pant, Common fied points of conditionally commuting maps, Fixed Point Theory, 11 (2010), 113-118.

[22] B.E. Rhoades, Some theorems on weakly contractive maps, Nonlinear Anal., 47 (2001), 2683-2693. doi: 10.1016/S0362-546X(01)00388-1

[23] M. Sarwar, R. Badshah-e, Some unique fixed point theorems in multiplicative metric space, ArXiv:1410.3384v2 [matn.GM] (2014), 19 pages.

[24] K.P.R. Sastry, S.V.R. Naidu, G.V.R. Babu, G.A. Naidu, Generalization of common fixed point theorems for weakly commuting mappings by altering distances, Tamkang J. Math., 31 (2000), 243-250.

[25] Q. Zhang, Y. Song, Fixed point theory for generalized $\psi$-weak contractions, Appl. Math. Lett., 22 (2009), 75-78. doi: 10.1016/j.aml.2008.02.007 
